\title{
Core-level interatomic Coulombic decay in van der Waals clusters
}

\author{
Andreas Hans $\odot,{ }^{1,2, *}$ Catmarna Küstner-Wetekam, ${ }^{1}$ Philipp Schmidt, ${ }^{1}$ Christian Ozga,,${ }^{1}$ Xaver Holzapfel,,${ }^{1}$ Huda Otto, ${ }^{1}$ \\ Christina Zindel, ${ }^{1}$ Clemens Richter $\odot,{ }^{3}$ Lorenz S. Cederbaum, ${ }^{4}$ Arno Ehresmann, ${ }^{1}$ Uwe Hergenhahn $\odot,{ }^{3,5}, \dagger$ \\ Nikolai V. Kryzhevoi $\odot{ }^{4, 末}$ and André Knie ${ }^{1}{ }^{1, \S}$ \\ ${ }^{1}$ Universität Kassel, Institut für Physik und CINSaT, Heinrich-Plett-Straße 40, 34132 Kassel, Germany \\ ${ }^{2}$ Nano and Molecular Systems Research Unit, Faculty of Science, University of Oulu, P.O. Box 3000, 90014 Oulu, Finland \\ ${ }^{3}$ Leibniz-Institut für Oberflächenmodifizierung (IOM), Permoserstraße 15, 04318 Leipzig, Germany \\ ${ }^{4}$ Theoretische Chemie, Physikalisch-Chemisches Institut, Universität Heidelberg, Im Neuenheimer Feld 229, 69120 Heidelberg, Germany \\ ${ }^{5}$ Fritz-Haber-Institut der Max-Planck-Gesellschaft, Faradayweg 4-6, D-14195 Berlin, Germany
}

(Received 20 June 2019; published 21 January 2020)

\begin{abstract}
We report on the experimental observation of the direct decay of a core vacancy in van der Waals clusters by emission of a fast electron from a neighboring atom. The process can be regarded as an interatomic Coulombic decay of core holes (core-level ICD). We identify it unambiguously by electron-electron and electron-electronphoton coincidence spectroscopy of the decay of $2 p$ vacancies in Ar clusters. While several earlier works reported the absence of this channel, we find core-level ICD to be of considerable significance and quantify the branching ratio of this nonlocal electron emission to conventional local Auger decay as $(0.8 \pm 0.2) \%$. Our results are supported by calculations on smaller clusters and show a reasonable agreement. This report on a successfully performed electron-electron-photon coincidence experiment provides a perspective for explorations of matter exposed to ionizing radiation. The observed core-level ICD is proposed to be of general importance for studies on charge redistribution after core-level photoionization where van der Waals clusters are often used as prototype systems.
\end{abstract}

DOI: 10.1103/PhysRevResearch.2.012022

The decay of electronic vacancies is among the most fundamental secondary processes induced by photoionization and therefore relevant for many fields of physics and chemistry. Except for vacancies in the outermost valence orbitals, in general an isolated ionized atom or molecule may decay either radiatively with emission of fluorescence radiation or, if the energy stored in the vacancy is above the second ionization energy, by emission of secondary Auger electrons [1,2].

The situation changes if an ion has neighbors. As first predicted theoretically [3] and later observed experimentally [4,5], an electronic decay mechanism called interatomic Coulombic decay (ICD) may be operational here (see also reviews [6-8]). While Auger decay occurs locally in the ion increasing its charge, ICD also involves the ion's environment, which gets ionized in turn. The charge created in ICD separates between different monomers. ICD processes are, in general, very fast. They quench fluorescence [9] and, if the number of monomers participating in ICD is sufficiently

\footnotetext{
*hans@physik.uni-kassel.de

${ }^{\dagger}$ Also at Max-Planck-Institut für Plasmaphysik, Wendelsteinstraße 1, 17491 Greifswald, Germany.

${ }^{\ddagger}$ nikolai.kryzhevoi@pci.uni-heidelberg.de

§nie@physik.uni-kassel.de

Published by the American Physical Society under the terms of the Creative Commons Attribution 4.0 International license. Further distribution of this work must maintain attribution to the author(s) and the published article's title, journal citation, and DOI.
}

large, may even compete with Auger decay [10]. In the related electron transfer mediated decay (ETMD), an electron from the environment is transferred to the ion and the environment gets ionized [11]. In contrast to ICD, ETMD neutralizes one charge of the initial ion and transfers two charge units to the environment.

In systems with a core vacancy, ICD or ETMD usually attracts attention as one part of various cascades starting with Auger transitions [12-17]. Yet, core vacancies can also directly decay by ICD. These so-called core-level ICD processes were recently studied both theoretically and experimentally in aqueous solutions and hydrogen-bound clusters using electron spectroscopy. They manifest themselves as shoulders or separate peaks on the high-kinetic-energy side of the local Auger peaks [18-24].

Although such processes were also predicted in van der Waals bound solid Ar, their rates were estimated to be extremely small (about seven orders of magnitude smaller than the local Auger decay rates), suggesting that these processes are elusive [25]. Indeed, experimental attempts failed to observe core-level ICD following $2 p$ ionization in Ar dimers [26] and large Ar clusters [27]. However, since noble gas clusters are commonly used as prototype systems in studies on interaction of matter with ionizing radiation, the direct nonlocal decay of core vacancies may have immediate implications. Especially in the emerging field of $x$-ray imaging with high-intensity free electron laser (FEL) radiation, processes like core-level ICD have not yet been considered to contribute to charge distribution and nanoplasma formation [28,29]. 

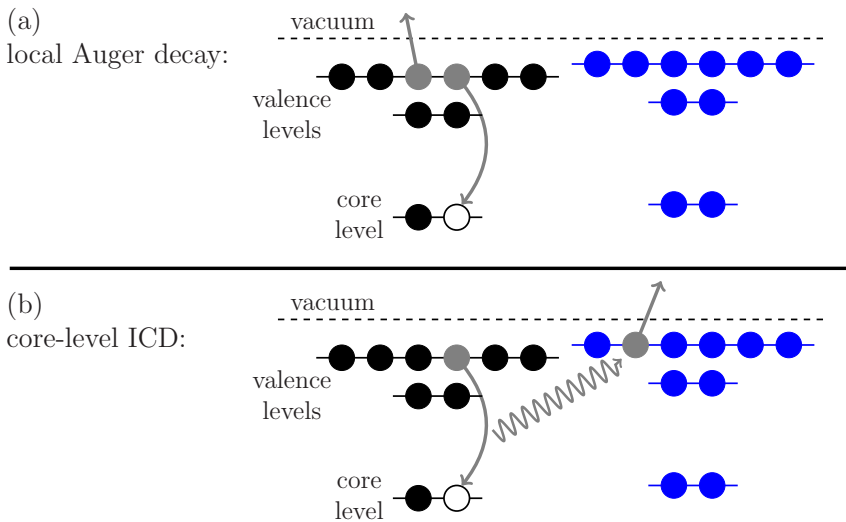

FIG. 1. Schematic representation of possible electronic decays in clusters. (a) Local Auger decay: a core vacancy is filled by a valence electron and another valence electron is emitted from the same atom. (b) Core-level ICD: a valence electron from the same atom fills the core vacancy and the released energy is transferred to a neighboring atom thereby ionizing it.

Here, we unambiguously demonstrate the occurrence of core-level ICD in van der Waals systems by presenting an observation of these processes in $2 p$ core-ionized Ar clusters. This observation became possible by the application of highly sensitive electron-electron and electron-electronphoton coincidence spectroscopies. The experimental results are supported by theoretical calculations.

In the present experiment, the $2 p$ vacancies were created by photoionization. The dominant relaxation channel of a $2 p$ hole is the Auger decay to localized one-site dicationic states with two outermost $3 p$ vacancies [Fig. 1(a)]:

$$
\operatorname{Ar}^{+}\left(2 p^{-1}\right) \operatorname{Ar}_{n-1} \underset{\text { Auger }}{\stackrel{\text { local }}{\longrightarrow}} \operatorname{Ar}^{2+}\left(3 p^{-2}\right) \operatorname{Ar}_{n-1}+e_{\text {Auger }}^{-} \text {. }
$$

As presented below in detail, also direct core-level ICD is possible in clusters, leading to two-site delocalized dicationic states [Fig. 1(b)]:

$$
\operatorname{Ar}^{+}\left(2 p^{-1}\right) \operatorname{Ar}_{n-1} \underset{\mathrm{ICD}}{\stackrel{\text { core }}{\longrightarrow}} \operatorname{Ar}^{+}\left(3 p^{-1}\right) \operatorname{Ar}^{+}\left(3 p^{-1}\right) \operatorname{Ar}_{n-2}+e_{\mathrm{ICD}}^{-}
$$

The experiment was performed at the BESSY II storage ring of the Helmholtz-Zentrum Berlin (HZB) using linearly polarized synchrotron radiation of the U49-2 PGM-1 beamline. A setup for electron-photon coincidence experiments as recently described in detail ([30], configuration A) was used, including the procedure of data acquisition and elimination of random coincidences. It combines a magnetic bottle timeof-flight electron spectrometer [31] and a mirror assembly for enhancing the solid angle of photon detection. A singlephoton detector equipped with a CsTe photocathode was used, sensitive for photons in the range from about 120 to $300 \mathrm{~nm}$ (about 4.0 to $10.4 \mathrm{eV}$ ) [32]. In the present experiment, the setup was used for the acquisition of coincidence events of two electrons and one photon.

Ar clusters were produced by supersonic expansion of Ar gas through a conical copper nozzle cooled with liquid nitrogen to temperatures between $105 \mathrm{~K}$ and $120 \mathrm{~K}$, corresponding to mean cluster sizes of $\langle N\rangle \approx 100$ and $\langle N\rangle \approx$ 50 [33]. Reference spectra of atomic Ar were taken using the same source at room temperature. Note that spectra of clusters inherently contain contributions from remaining free atoms. Spectra were recorded at exciting-photon energies $E=436 \mathrm{eV}$ and $E=449 \mathrm{eV}$. For further details on target preparation and the coincident detection of photoelectrons and Auger electrons see Ref. [33].

The experimental $L_{3} M M$ and $L_{2} M M$ Auger spectra of atomic Ar measured in coincidence with the respective $2 p_{3 / 2}$ and $2 p_{1 / 2}$ photoelectrons are shown in Fig. 2(a). They are separated by $2.2 \mathrm{eV}$ corresponding to the spin-orbit splitting of the $\operatorname{Ar} 2 p^{-1}$ core-hole state, and each component shows contributions from the ${ }^{3} P_{0,1,2},{ }^{1} D_{2}$, and ${ }^{1} S_{0}$ final dicationic states [34]. Auger electrons with lower kinetic energies (i.e., higher binding energy final states) do not reach the detector due to the retardation voltage applied [33]. Since the electron spectrometer is not well characterized for such high retardation voltages [31], some line shapes and relative intensities of the spectral contributions may deviate from the literature values [34].

Compared to the pure atomic spectra, the electron-electron coincidence Auger spectra of a partially condensed Ar cluster jet represented in Fig. 2(b) show two new structures: the prominent shoulders at the high-kinetic-energy side of the atomic peaks and additional weak peaks at even higher kinetic energies which are well separated from the main Auger lines and only visible in a magnified presentation. Both structures appear due to electronic processes in clusters: while the former originates from Auger decays, the latter is attributed to core-level ICD processes, as explained in detail below.

The atomic and cluster contributions cannot be separated in the spectra of Fig. 2(b) because the resolution achieved does not allow for a proper separation of photoelectrons from atoms and clusters. However, for the quantification of the branching ratio of core ICD to local Auger decay it is necessary to isolate the Auger spectra from clusters. Toward this end we used electron-photon and electron-electron-photon coincident methods to efficiently eliminate the atomic contribution from the spectra.

As demonstrated recently, no electron-photon coincidences can be observed in atomic Ar for Auger electrons corresponding to the $\operatorname{Ar}^{2+}\left(3 p^{-2}{ }^{3} P_{0,1,2},{ }^{1} D_{2}\right)$ final states [30]. A weak signal appears only for the $\operatorname{Ar}^{2+}\left(3 p^{-2}{ }^{1} S_{0}\right)$ state. The situation is, however, dramatically different in clusters. Here, all localized Auger $\mathrm{Ar}^{2+}\left(3 p^{-2}\right)$ states decay further by radiative charge transfer $(\mathrm{RCT})[26,35]$ :

$$
\operatorname{Ar}^{2+}\left(3 p^{-2}\right) \operatorname{Ar}_{n-1} \rightarrow \operatorname{Ar}^{+}\left(3 p^{-1}\right) \operatorname{Ar}^{+}\left(3 p^{-1}\right) \operatorname{Ar}_{n-2}+h v_{\mathrm{RCT}} .
$$

The energies of the emitted RCT photons can be roughly estimated from the difference between the potential energy curves of the dicationic states at the approximate interatomic distance $(\sim 2.7 \AA)$ at which RCT takes place in $\mathrm{Ar}_{2}[26,35]$. These energies are about $6.6 \mathrm{eV}$ and $8.3 \mathrm{eV}$ for the ${ }^{3} P_{0,1,2}$ and ${ }^{1} D_{2}$ states, respectively, which are well within the sensitivity range of the photon detector. RCT processes involving the ${ }^{1} S_{0}$ states result however in the emission of photons of energies around $10.7 \mathrm{eV}$, for which the efficiency of the detector 


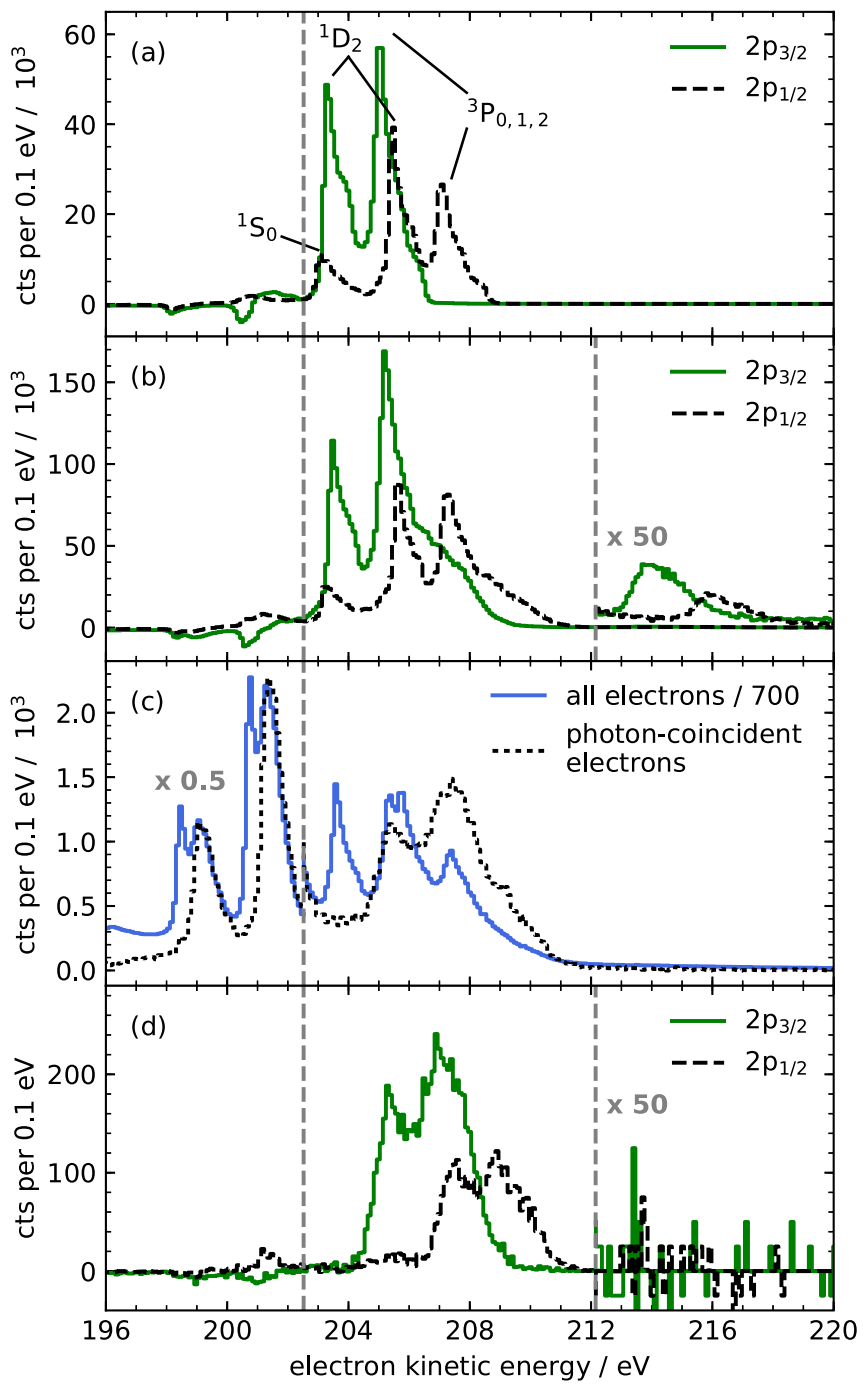

FIG. 2. Electron spectra after $2 p$ ionization of Ar recorded at an exciting-photon energy of $E=449 \mathrm{eV}(-190 \mathrm{~V}$ retarding potential). Units of the intensity axis are coincidence counts per $0.1 \mathrm{eV}$ energy bin. (a) Auger spectra of atomic Ar separated according to the spin-orbit components measured in coincidence with the respective photoelectrons (see text). (b) Analogously measured Auger spectra of a partially condensed Ar cluster jet (120 K nozzle temperature). Note the peaks appearing on the high kinetic energy side are missing in the atomic spectra. (c) Spectrum of photon-coincident electrons compared to the overall electron spectrum obtained without coincidences, all for the Ar cluster jet. The peaks seen on the left side of the vertical dashed line are due to the photoelectrons. (d) Pure cluster Auger spectra separated for $2 p_{3 / 2}$ and $2 p_{1 / 2}$ photoionization as obtained from electron-electron-photon coincidence spectra. No photon is emitted from the states corresponding to the high kinetic energy side shedding light on their different nature (see text).

is extremely low, and may not be detected in the present experiment.

In Fig. 2(c), the electron-photon coincidence spectrum for the Ar cluster jet is compared to the overall electron spectrum obtained without coincidences. These spectra have photoelectron peaks on the left side of the dashed line in the kinetic energy range between 197-202 eV, which are filtered out in all other spectra in Fig. 2 [33]. As seen from these peaks, the atomic contributions (the lower kinetic energy parts in the double-peak structures) are essentially removed by the electron-photon coincidences, and the respective photoncoincident spectrum can thus be regarded as the pure cluster spectrum. Yet, the contributions from the initial $2 p_{3 / 2}$ and $2 p_{1 / 2}$ photoionizations cannot be distinguished here because only one electron was measured in coincidence with the RCT photon. However, the two Auger components can be obtained separately by making use of electron-electron-photon coincidences enabling us to filter Auger spectra for emission of an RCT photon and either the $2 p_{3 / 2}$ or $2 p_{1 / 2}$ photoelectrons. These components are shown in Fig. 2(d). As explained above, there are no contributions from the ${ }^{1} S_{0}$ Auger final states in these spectra.

The LMM Auger spectra of Ar clusters have been studied in several previous works [26,27,36,37]. Pure cluster spectra have been obtained and thoroughly investigated in Ref. [36] with respect to cluster size, scattering, and postcollision interaction effects. The pure cluster Auger spectrum shown in Fig. 2(c) agrees well with them. Compared to previous studies, the considerable advance of the present work is the separation of the pure cluster Auger spectrum into the $L_{3} M M$ and $L_{2} M M$ components [Fig. 2(d)]. Both have similar structures and in particular the $L_{3} M M$ component is twice as intense as the $L_{2} M M$ one. Note that due to the coincidence condition imposed, the present spectra in Fig. 2(d) are free of influences of photoelectron recapture and scattering.

To support the assignment of the two weak peaks in the 212-218 eV kinetic energy region [Fig. 2(b)] to core-level ICD, we first consider their energy separation to the respective pure cluster Auger peaks. They should be related to the energy difference between one-site and two-site dicationic states in clusters. In $\mathrm{Ar}_{2}$, this energy difference is expected to be about $8 \mathrm{eV}$ [26], which is a little larger than the $7.1 \mathrm{eV}$ inferred from the current spectra. This discrepancy is likely associated with cluster size and polarization screening effects [38]: in larger clusters the energy separation between one-site and two-site dicationic states should decrease because of a larger polarization screening shift experienced by the former states.

The branching ratio of core-level ICD and local Auger processes is an important parameter for many applications. We have determined it as follows. For each of the $2 p_{1 / 2}$ and $2 p_{3 / 2}$ components, the pure atomic [Fig. 2(a)] and pure cluster [Fig. 2(d)] Auger spectra were added with individual variable scaling factors, until the best fit with the cluster jet Auger spectrum [Fig. 2(b)] was found. Then, the areas of the pure cluster Auger contribution and the ICD peak (including the scaling factors) were integrated and compared. Finally, we account for the absence of the ${ }^{1} S_{0}$ channel in the electronelectron-photon coincident spectra [Fig. 2(d)], which has an intensity on the order of $10 \%$ of the total $L_{2,3} M_{2,3} M_{2,3}$ area. The intensity of the local Auger decay was corrected by $10 \%$, yielding the final result for the core-level ICD to local Auger decay branching ratio of $(0.8 \pm 0.2) \%$.

To further support the above interpretation of the experimental spectra, we have calculated Auger spectra of several size-selected $\operatorname{Ar}_{n}(n=2-7,13,19)$ clusters. The cluster geometries were taken from the Cambridge Cluster Database [39]. In order to exclude the effect of different interatomic 


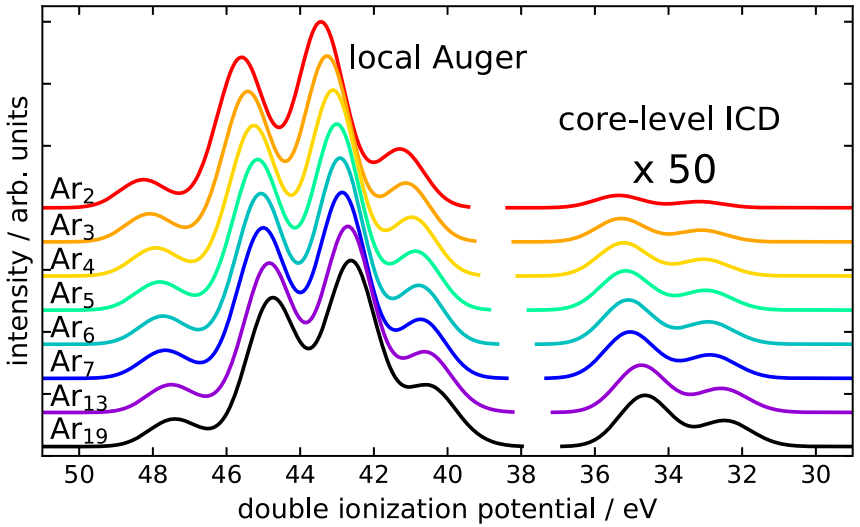

FIG. 3. Calculated averaged Auger spectra of Ar clusters of different sizes plotted on the double-ionization energy scale. All spectra exhibit two well-separated structures attributed to different decay processes: local Auger decay (higher DIPs) and core-level ICD (lower DIPs). The relative intensities of the core-level ICD peaks grow with increasing cluster size.

separations on the spectra computed in the present work, the same nearest-neighbor distances of $3.76 \AA$, corresponding to the $\mathrm{Ar}-\mathrm{Ar}$ bond in $\mathrm{Ar}_{2}$, were used in all clusters. The spectra were computed using a combination of the $a b$ initio secondorder algebraic diagrammatic construction [ADC(2)] method $[40,41]$ in conjunction with a reduced relativistic pseudopotential basis set [42], and the two-hole population analysis [43]. An overview of the main steps of the computational protocol is given in Ref. [33]. For more details, see previous publications [19,20,23].

The calculated spectra are plotted on the double-ionization potential (DIP) scale in Fig. 3 as a function of cluster size. The spectra are averaged over the number of atoms present at different sites in a cluster and normalized to the maximum intensity. Like the experimental spectra, the calculated ones exhibit two well-separated features. The high-energy and intense multipeak structure is attributed to Auger processes since all dicationic states contributing here are of a one-site character. In contrast, the low-energy and faint double-peak structure (missing in an isolated atom) entirely contains delocalized dicationic states and is thus assigned to interatomic decay processes. Both spectral structures move toward lower DIPs with increasing cluster size due to polarization screening [38]. The shift is, however, larger for the localized states so that the energy gap between these two spectral structures gradually decreases, corroborating the above supposition. Besides the energy shifts, also the intensity ratio of the ICD and Auger peaks changes notably. In $\mathrm{Ar}_{2}$ it is only $0.09 \%$, explaining the failure to observe core-level ICD in experiments on dimers earlier [26], but reaches $0.35 \%$ and $0.47 \%$ in $\mathrm{Ar}_{13}$ and $\mathrm{Ar}_{19}$, respectively.

The deviation between the theoretical core-level ICD to local Auger branching ratios and the experimental value can be explained by the different sizes of the experimental and theoretical clusters. In the former systems, the number of bulk atoms possessing a complete coordination shell of twelve neighbors is appreciable, whereas only a very few such atoms are present in the latter clusters and the rest

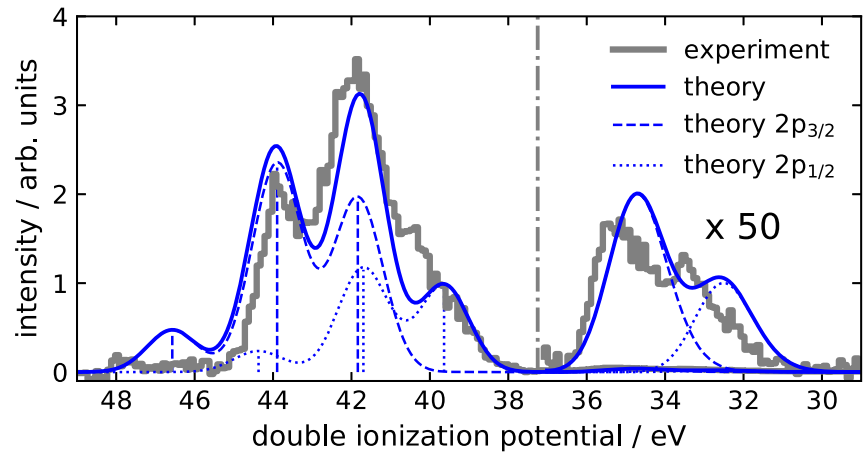

FIG. 4. Comparison of the experimental spectrum with the theoretical spectrum of the central atom in an $\operatorname{Ar}_{13}$ cluster. The experimental spectrum is composed of results from electronelectron-photon coincidences (local Auger) and electron-electron coincidences (core-level ICD) to higher and lower DIPs of the vertical dash-dotted line, respectively (see Fig. 2 and text).

consist of "surface" monomers with incomplete coordination shells. The effect of bulk atoms on Auger spectra is, however, remarkable as can be inferred from Fig. 4 comparing the experimental pure cluster spectrum with a theoretical one obtained for the central "bulk" atom in $\mathrm{Ar}_{13}$. Both spectra are plotted on the DIP scale such that the positions of the main maxima composed of the $L_{2} M M{ }^{1} D_{2}$ and $L_{3} M M{ }^{3} P_{0,1,2}$ peaks coincide and are normalized to the area of the Auger states (38-48 eV). The experimental spectrum is composed of results from electron-electron-photon coincidences (local Auger region) and electron-electron coincidences (core-level ICD region).

The spectra overall agree well. A perfect agreement is not expected since the experimental spectrum originates from a wide size distribution of clusters and different sites within each cluster, which cannot be reproduced by the calculations for size-selected clusters. Qualitatively, in the DIP region above $43 \mathrm{eV}$ the experimental spectrum is lacking the contribution from the ${ }^{1} S_{0}$ states (see above). The energy gap between the Auger and core-level ICD peaks is somewhat smaller in the experimental spectrum, suggesting that the polarization screening due to Ar atoms beyond the first coordination shell is not negligible. The theoretical core-level ICD to Auger branching ratio is $0.98 \%$ for the central atom in $\mathrm{Ar}_{13}$ and thus larger than for the experiment. However, the experimental value lies reasonably between the values of the central atom in $\mathrm{Ar}_{13}$ and the total $\mathrm{Ar}_{13}$ cluster, which contains mostly surface atoms.

In conclusion, we have observed core-level ICD processes in van der Waals bound clusters by applying electron-electron and electron-electron-photon coincidence spectroscopy. The efficiency of these processes has been found to be about $0.8 \%$ of the Auger decay which is smaller than in hydrogen-bonded systems but not negligible as predicted earlier for van der Waals systems [25]. Our theoretical calculations support the experimental observation. They also reveal the sensitivity of core-level ICD peaks to the local environment, which can be used for probing the geometric and electronic structures of various clusters, surfaces, and interfaces. Mixed Xe-Ar clusters could be particularly attractive systems for a further 
study. Due to the proximity of one-site and two-site dicationic states in these clusters [44], core-level ICD peaks may partially overlap with Auger peaks acquiring thus considerable intensity through borrowing it from the Auger states [22]. In addition, core-level ETMD may be observed there. In strong contrast to homonuclear clusters considered in the present work, the energies of ETMD states and the created ions in mixed systems are distinguishable from those produced in ICD and Auger processes. Recently, core-level ETMD processes were observed in aqueous solutions $[45,46]$. Our report is on a successful electron-electron-photon coincidence experiment. We emphasize that this experimental method is by no means restricted to applications on core-level ICD, but generally enriches the toolbox of experimental atomic, molecular, and cluster physics.

We thank HZB for beamtime allocation and the BESSY II staff for assistance. This work was funded by the Deutsche Forschungsgemeinschaft (DFG) (Project No. 328961117 . SFB 1319 ELCH and Research Unit FOR 1789). L.S.C. is grateful to the European Research Council (Advanced Investigator Grant No. 692657) for financial support.
[1] L. Meitner, Über die $\beta$-strahl-spektra und ihren zusammenhang mit der $\gamma$-strahlung, Z. Phys. 11, 35 (1922).

[2] P. Auger, Sur l'effet photoélectrique composé, J. Phys. Radium 6, 205 (1925).

[3] L. S. Cederbaum, J. Zobeley, and F. Tarantelli, Giant Intermolecular Decay and Fragmentation of Clusters, Phys. Rev. Lett. 79, 4778 (1997).

[4] S. Marburger, O. Kugeler, U. Hergenhahn, and T. Möller, Experimental Evidence for Interatomic Coulombic Decay in $\mathrm{Ne}$ Clusters, Phys. Rev. Lett. 90, 203401 (2003).

[5] T. Jahnke, A. Czasch, M. S. Schöffler, S. Schössler, A. Knapp, M. Käsz, J. Titze, C. Wimmer, K. Kreidi, R. E. Grisenti, A. Staudte, O. Jagutzki, U. Hergenhahn, H. Schmidt-Böcking, and R. Dörner, Experimental Observation of Interatomic Coulombic Decay in Neon Dimers, Phys. Rev. Lett. 93, 163401 (2004).

[6] V. Averbukh, Ph. V. Demekhin, P. Kolorenč, S. Scheit, S. D. Stoychev, A. I. Kuleff, Y.-C. Chiang, K. Gokhberg, S. Kopelke, N. Sisourat, and L. S. Cederbaum, Interatomic electronic decay processes in singly and multiply ionized clusters, J. Electron Spectrosc. Relat. Phenom. 183, 36 (2011).

[7] U. Hergenhahn, Interatomic and intermolecular Coulombic decay: The early years, J. Electron Spectrosc. Relat. Phenom. 184, 78 (2011).

[8] T. Jahnke, Interatomic and intermolecular Coulombic decay: The coming of age story, J. Phys. B: At., Mol. Opt. Phys. 48, 082001 (2015).

[9] A. Hans, P. Schmidt, C. Ozga, C. Richter, H. Otto, X. Holzapfel, G. Hartmann, A. Ehresmann, U. Hergenhahn, and A. Knie, Efficient fluorescence quenching by distant production of a free electron, J. Phys. Chem. Lett. 10, 1078 (2019).

[10] N. V. Kryzhevoi, V. Averbukh, and L. S. Cederbaum, High activity of helium droplets following ionization of systems inside those droplets, Phys. Rev. B 76, 094513 (2007).

[11] J. Zobeley, R. Santra, and L. S. Cederbaum, Electronic decay in weakly bound heteroclusters: Energy transfer versus electron transfer, J. Chem. Phys. 115, 5076 (2001).

[12] R. Santra and L. S. Cederbaum, Coulombic Energy Transfer and Triple Ionization in Clusters, Phys. Rev. Lett. 90, 153401 (2003).

[13] Y. Morishita, X.-J. Liu, N. Saito, T. Lischke, M. Kato, G. Prümper, M. Oura, H. Yamaoka, Y. Tamenori, I. H. Suzuki, and K. Ueda, Experimental Evidence of Interatomic Coulombic Decay from the Auger Final States in Argon Dimers, Phys. Rev. Lett. 96, 243402 (2006).
[14] K. Gokhberg, P. Kolorenč, A. I. Kuleff, and L. S. Cederbaum, Site- and energy-selective slow-electron production through intermolecular Coulombic decay, Nature (London) 505, 661 (2014).

[15] F. Trinter, M. S. Schöffler, H.-K. Kim, F. P. Sturm, K. Cole, N. Neumann, A. Vredenborg, J. Williams, I. Bocharova, R. Guillemin, M. Simon, A. Belkacem, A. L. Landers, Th. Weber, H. Schmidt-Böcking, R. Dörner, and T. Jahnke, Resonant Auger decay driving intermolecular Coulombic decay in molecular dimers, Nature (London) 505, 664 (2014).

[16] V. Stumpf, K. Gokhberg, and L. S. Cederbaum, The role of metal ions in x-ray-induced photochemistry, Nat. Chem. 8, 237 (2016).

[17] H. Fukuzawa, Y. Li, D. You, Y. Sakakibara, S. Yamada, Y. Ito, T. Takanashi, M. Oura, N. Saito, and K. Ueda, Lowenergy-electron production after $2 p$ ionization of argon clusters, Phys. Rev. A 99, 042505 (2019).

[18] E. F. Aziz, N. Ottosson, M. Faubel, I. V. Hertel, and B. Winter, Interaction between liquid water and hydroxide revealed by core-hole de-excitation, Nature (London) 455, 89 (2008).

[19] W. Pokapanich, H. Bergersen, I. L. Bradeanu, R. R. T. Marinho, A. Lindblad, S. Legendre, A. Rosso, S. Svensson, O. Björneholm, M. Tchaplyguine, G. Ohrwall, N. V. Kryzhevoi, and L. S. Cederbaum, Auger electron spectroscopy as a probe of the solution of aqueous ions, J. Am. Chem. Soc. 131, 7264 (2009).

[20] W. Pokapanich, N. V. Kryzhevoi, N. Ottosson, S. Svensson, L. S. Cederbaum, G. Öhrwall, and O. Björneholm, Ionic-charge dependence of the intermolecular Coulombic decay time scale for aqueous ions probed by the core-hole clock, J. Am. Chem. Soc. 133, 13430 (2011).

[21] N. V. Kryzhevoi and L. S. Cederbaum, Nonlocal effects in the core ionization and Auger spectra of small ammonia clusters, J. Phys. Chem. B 115, 5441 (2011).

[22] N. V. Kryzhevoi and L. S. Cederbaum, Exploring protonation and deprotonation effects with Auger electron spectroscopy, J. Phys. Chem. Lett. 3, 2733 (2012).

[23] P. Slavíček, B. Winter, L. S. Cederbaum, and N. V. Kryzhevoi, Proton-transfer mediated enhancement of nonlocal electronic relaxation processes in $\mathrm{x}$-ray irradiated liquid water, J. Am. Chem. Soc. 136, 18170 (2014).

[24] N. V. Kryzhevoi, Microhydration of LiOH: Insight from electronic decays of core-ionized states, J. Chem. Phys. 144, 244302 (2016). 
[25] J. A. D. Matthew and Y. Komninos, Transition rates for interatomic Auger processes, Surf. Sci. 53, 716 (1975).

[26] N. Saito, Y. Morishita, I. H. Suzuki, S. D. Stoychev, A. I. Kuleff, L. S. Cederbaum, X.-J. Liu, H. Fukuzawa, G. Prümper, and K. Ueda, Evidence of radiative charge transfer in argon dimers, Chem. Phys. Lett. 441, 16 (2007).

[27] M. Lundwall, M. Tchaplyguine, G. Öhrwall, A. Lindblad, S. Peredkov, T. Rander, S. Svensson, and O. Björneholm, Enhanced surface sensitivity in AES relative to XPS observed in free argon clusters, Surf. Sci. 594, 12 (2005).

[28] T. Tachibana, Z. Jurek, H. Fukuzawa, K. Motomura, K. Nagaya, S. Wada, P. Johnsson, M. Siano, S. Mondal, Y. Ito, M. Kimura, T. Sakai, K. Matsunami, H. Hayashita, J. Kajikawa, X.-J. Liu, E. Robert, C. Miron, R. Feifel, J. P. Marangos, K. Tono, Y. Inubushi, M. Yabashi, S.-K. Son, B. Ziaja, M. Yao, R. Santra, and K. Ueda, Nanoplasma formation by high intensity hard X-rays, Sci. Rep. 5, 10977 (2015).

[29] Y. Kumagai, H. Fukuzawa, K. Motomura, D. Iablonskyi, K. Nagaya, S. Wada, Y. Ito, T. Takanashi, Y. Sakakibara, D. You, T. Nishiyama, K. Asa, Y. Sato, T. Umemoto, K. Kariyazono, E. Kukk, K. Kooser, C. Nicolas, C. Miron, T. Asavei, L. Neagu, M. S. Schöffler, G. Kastirke, X.-J. Liu, S. Owada, T. Katayama, T. Togashi, K. Tono, M. Yabashi, N. V. Golubev, K. Gokhberg, L. S. Cederbaum, A. I. Kuleff, and K. Ueda, Following the Birth of A Nanoplasma Produced by an Ultrashort Hard-X-Ray Laser in Xenon Clusters, Phys. Rev. X 8, 031034 (2018).

[30] A. Hans, C. Ozga, P. Schmidt, G. Hartmann, A. Nehls, P. Wenzel, C. Richter, C. Lant, X. Holzapfel, J. H. Viehmann, U. Hergenhahn, A. Ehresmann, and A. Knie, Setup for multicoincidence experiments of EUV to VIS photons and charged particles: The solid angle maximization approach, Rev. Sci. Instrum. 90, 093104 (2019).

[31] M. Mucke, M. Förstel, T. Lischke, T. Arion, A. M. Bradshaw, and U. Hergenhahn, Performance of a short "magnetic bottle" electron spectrometer, Rev. Sci. Instrum. 83, 063106 (2012).

[32] A. Hans, P. Schmidt, C. Ozga, G. Hartmann, X. Holzapfel, A. Ehresmann, and A. Knie, Extreme ultraviolet to visible dispersed single photon detection for highly sensitive sensing of fundamental processes in diverse samples, Materials 11, 869 (2018).

[33] See Supplemental Material at http://link.aps.org/supplemental/ 10.1103/PhysRevResearch.2.012022 for details on target preparation, coincidence detection, and computational details.

[34] L. O. Werme, T. Bergmark, and K. Siegbahn, The $L_{2,3} M M$ Auger spectrum of argon, Phys. Scr. 8, 149 (1973).
[35] S. D. Stoychev, A. I. Kuleff, F. Tarantelli, and L. S. Cederbaum, On the doubly ionized states of $\mathrm{Ar}_{2}$ and their intra- and interatomic decay to $\mathrm{Ar}_{2}^{3+}$, J. Chem. Phys. 128, 014307 (2008).

[36] M. Lundwall, A. Lindblad, H. Bergersen, T. Rander, G. Öhrwall, M. Tchaplyguine, S. Peredkov, S. Svensson, and O. Björneholm, Photon energy dependent intensity variations observed in Auger spectra of free argon clusters, J. Phys. B: At., Mol. Opt. Phys. 39, 3321 (2006).

[37] M. Lundwall, A. Lindblad, G. Öhrwall, S. Svensson, and O. Björneholm, Neighbor-induced photoelectron recapture in argon clusters: A photon-energy-dependent study of Auger spectra, Phys. Rev. A 78, 065201 (2008).

[38] O. Björneholm, F. Federmann, F. Fössing, and T. Möller, Core Level Photoelectron and X-Ray Absorption Spectroscopy of Free Argon Clusters: Size-Dependent Energy Shifts and Determination of Surface Atom Coordination, Phys. Rev. Lett. 74, 3017 (1995).

[39] D. J. Wales, J. P. K. Doye, A. Dullweber, M. P. Hodges, F. Y. Naumkin, F. Calvo, J. Hernández-Rojas, and T. F. Middleton, The Cambridge Cluster Database, http://www-wales.ch.cam.ac. $\mathrm{uk} / \sim$ wales/CCD/AzizAr.html.

[40] J. Schirmer and A. Barth, Higher-order approximations for the particle-particle propagator, Z. Phys. 317, 267 (1984).

[41] F. Tarantelli, The calculation of molecular double ionization spectra by Green's functions, Chem. Phys. 329, 11 (2006).

[42] W. Küchle, M. Dolg, H. Stoll, and H. Preuss, Ab initio pseudopotentials for Hg through Rn, Mol. Phys. 74, 1245 (1991).

[43] F. Tarantelli, A. Sgamellotti, and L. S. Cederbaum, Many dicationic states and two-hole population analysis as a bridge to Auger spectra: Strong localization phenomena in $\mathrm{BF}_{3}$, J. Chem. Phys. 94, 523 (1991).

[44] E. Fasshauer, N. V. Kryzhevoi, and M. Pernpointner, Possible electronic decay channels in the ionization spectra of small clusters composed of Ar and Xe: A four-component relativistic treatment, J. Chem. Phys. 133, 014303 (2010).

[45] I. Unger, R. Seidel, S. Thürmer, M. N. Pohl, E. F. Aziz, L. S Cederbaum, E. Muchová, P. Slavíček, B. Winter, and N. V. Kryzhevoi, Observation of electron-transfer-mediated decay in aqueous solution, Nat. Chem. 9, 708 (2017).

[46] M. N. Pohl, C. Richter, E. Lugovoy, R. Seidel, P. Slavíček, E. F. Aziz, B. Abel, B. Winter, and U. Hergenhahn, Sensitivity of electron transfer mediated decay to ion pairing, J. Phys. Chem. B 121, 7709 (2017). 